abnormal systemic branches and a huge RCA aneurysm in adulthood.

\section{References}

1. Bland EF, White PD, Garland J. Congenital anomalies of the coronary arteries: report of an unusual case associated with cardiac hypertrophy. Am Heart J. 1933;8:787-801

2. Wesselhoeft H, Fawcett JS, Johnson AL. Anomalous origin of the left coronary artery from the pulmonary trunk. Its clinical spectrum, pathology, and pathophysiology based on a review of 140 cases with seven further cases. Circulation. 1968;38:403-25.
3. Koike K, Musewe NN, Smallhorn JF, Freedom RM. Distinguishing between anomalous origin of the left coronary artery from the pulmonary trunk and dilated cardiomyopathy: role of echocardiographic measurement of the right coronary artery diameter. Br Heart J. 1989;61: 192-7.

4. Arsan S, Naseri E, Keser N. An adult case of Bland White Garland syndrome with huge right coronary aneurysm. Ann Thorac Surg. 1999; 68:1832-3.

5. Barbetakis N, Efstathiou A, Papagiannopoulou P, Soulountsi V, Fessatidis I. A long-term survivor of Bland-White-Garland syndrome with systemic collateral supply: a case report and review of the literature. BMC Surg. 2005;5:23.

\title{
Acute intramural hematoma of the aorta as a cause of positive fluorodeoxyglucose positron emission tomography/computed tomography
}

Alex Ryan, MD, Barry McCook, MD, Biatta Sholosh, MD, Daniel A. Pryma, MD, Eric Jablonowski, Carl Fuhrman, MD, and Todd M. Blodgett, MD, Pittsburgh, Pa

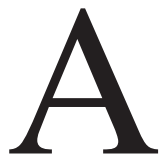

cute intramural hematoma (IMH) is a process characterized by aortic wall hemorrhage without intimal disruption. It has recently been added to the list of acute aortic syndromes (AASs), which also includes aortic dissection (AD), penetrating atherosclerotic ulcer (PAU), aneurysmal leak, and traumatic transection. IMH accounts for roughly $10 \%$ to $30 \%$ of AAS cases. ${ }^{1}$

The hemorrhage of IMH is said to arise from (1) rupture of the vasa vasorum in the adventitia of the aortic wall and (2) progression of a PAU. PAU is largely a disease of the descending aorta, making vasa vasorum rupture the major cause of upper aorta, type A IMH. ${ }^{2}$ These type A lesions usually occur within a few centimeters of the aortic valve on the lateral wall of the ascending aorta. The wall failure in IMH occurs nearer the adventitia than does the failure of $\mathrm{AD}$, which helps explain both the higher incidence of rupture $(35 \%)^{3}$ and the lower likelihood of reentry, which is the cause of false lumen flow in AD. Progression to AD is nevertheless not uncommon during follow-up of IMH cases, with a prevalence of $12 \%{ }^{4}$

Clinical presentation of AAS classically involves sudden back pain, chest pain, or both, and it generally presents in hypertensive patients $(85 \%) .{ }^{5}$ AAS can be mistaken for myocardial infarction with cardiogenic shock, especially when rupture leads to tamponade. Traumatic deceleration injuries can cause IMH through in-

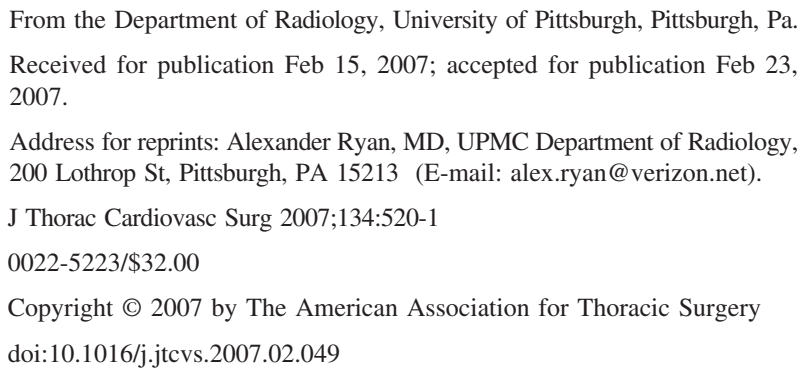

complete traumatic aortic rupture. Misdiagnosis in these patients is often fatal, making early intervention essential.

IMH can be diagnosed using computed tomography (CT) and usually appears as a crescent-shaped area that is higher attenuation than intraluminal blood on noncontrast images (Figure 1). To our knowledge, IMH has not been described in the literature as a cause of fluorodeoxyglucose (FDG) uptake on FDG positron emission tomography (PET) or PET/CT.

\section{Clinical Summary}

Initial PET/CT imaging was performed on a 70-year-old woman for staging of colorectal cancer. PET and diagnostic unenhanced CT images were acquired from the base of the skull to the proximal thighs on a GE Discovery LS PET/CT (GE Medical Systems, Waukesha, Wis) approximately 60 minutes after intravenous injection of $540 \mathrm{MBq}$ of FDG. The PET image showed a subtle tiny focus of FDG activity in the aorta; however, no correlative CT abnormality could be identified to make a definitive diagnosis, and the uptake was attributed to atherosclerotic change (Figure 2).

Approximately 6 months later, PET/CT was performed for restaging after partial colectomy. Contrast CT was not obtained, but on unenhanced CT, 2 new findings were found: mild aneurysmal dilation of the ascending aorta and a subtle but enlarging, high-attenuation, crescent-shaped area along the lateral aortic wall.

On the PET portion of the examination, findings included a crescent-shaped area of intense increased FDG activity along the left lateral wall of the ascending aorta with a maximum standardized uptake value (SUV) of 6.4. These findings confirmed the diagnosis of acute IMH. The focus noted in the earlier study was now recognized as a very early stage of this patient's IMH.

\section{Discussion}

Only recently was IMH differentiated from $\mathrm{AD}$ as an entity with its own characteristics. It has in common with $\mathrm{AD}$ its relation to hypertension and the unsolved dilemma regarding whether to 

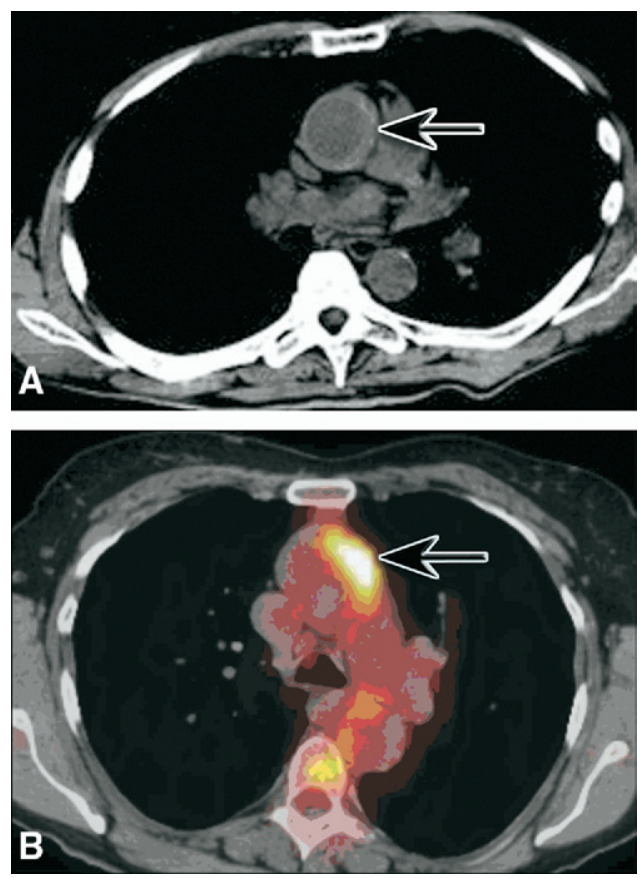

Figure 1. Select computed tomographic (CT) and positron emission tomographic (PET)/CT images showing aortic intramural hematoma. A, Axial CT shows a circumferential area of high attenuation compared with the intraluminal contents of the inner aortic lumen. B, A fused PET/CT image demonstrates high uptake in aortic wall after progression of hematoma.

perform early surgical intervention or to treat medically. The IMH population differs from the AD population in that patients tend to be older and more often female, and no association with Marfan's syndrome has been described. IMH also carries a higher risk of cardiac tamponade at presentation than $\mathrm{AD}$ or PAU. ${ }^{1}$

The burden on the radiologist is to differentiate IMH from other aortic syndromes and to assess the risk of progression in a given case of IMH. The prerequisite finding for IMH is the presence of a high-attenuation crescentic area along the aortic wall without a false lumen. Contrast CT has become the gold standard for this diagnosis, although noncontrast CT can be very specific for IMH when a high-attenuation crescent-shaped area of hematoma is seen, as with our patient. Intimal disruption and penetrating ulcers argue against the diagnosis of IMH. This can present a challenge in some cases because small intimal disruptions can be overlooked not only by the radiologist but also during surgical intervention and autopsy as well. Difficulty can also arise in differentiating IMH from intraluminal thrombus. Intraluminal thrombus tends to be localized and found within a dilated aorta, whereas IMH often extends over a longer segment of a nondilated aorta.

Clinically, the challenge with IMH is to determine whether a patient with an acute IMH will resolve or progress to rupture. The
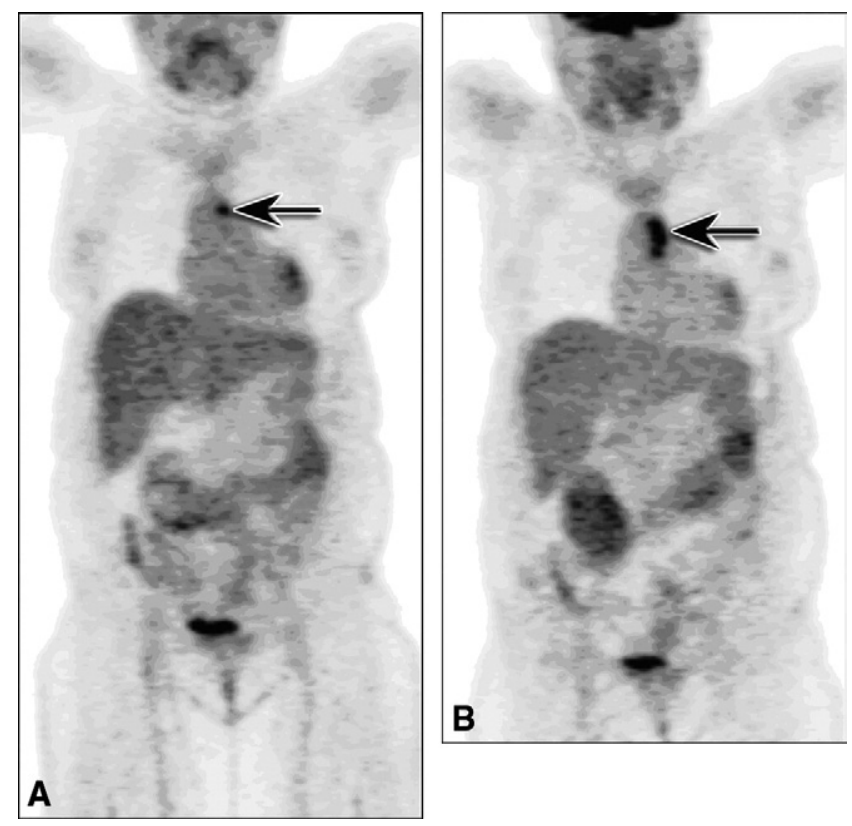

Figure 2. Coronal positron emission tomographic (PET) images showing aortic intramural hematoma. A, Coronal PET image from the initial PET/computed tomographic (CT) scan demonstrates a small focus of abnormal FDG activity in the aortic wall (arrow) consistent with early presentation of intramural hematoma. B, Coronal image from a follow-up PET/CT performed 6 months later shows progression of abnormal uptake caused by intramural hematoma (arrow).

surgical options are not straightforward; for example, stent grafting is often impossible because of possible occlusion of arch vessels in the vicinity of the IMH. It is important to remember that contrast CT is the diagnostic modality of choice, and prompt diagnosis is essential to selecting an appropriate treatment strategy.

\section{References}

1. Song JK, Kang SJ, Song JM, Kang DH, Song H, Chung CH, et al. Factors associated with in-hospital mortality in patients with acute aortic syndrome involving the ascending aorta. Int J Cardiol. 2007;115: 14-8.

2. Nakamura K, Onitsuka T, Yano M, Yano Y, Matsuyama M, Kojima K. Clinical analysis of acute type A intramural hematoma: comparison between two different pathophysiological types. Ann Thorac Surg. 2006;81:1587-92.

3. Ahmad F, Cheshire N, Hamady M. Acute aortic syndrome: pathology and therapeutic strategies. Postgrad Med J. 2006;82:305-12.

4. Chiles C, Carr JJ. Vascular diseases of the thorax: evaluation with multidetector CT. Radiol Clin North Am. 2005;43:543-69, viii.

5. Sueyoshi E, Sakamoto T, Uetani M, Matsuoka Y. CT analysis of the growth rate of aortic diameter affected by acute type B intramural hematoma. AJR Am J Roentgenol. 2006;186(suppl 2):S414-20. 